\title{
STANT MANIBUS ARAE. I MANES NELL'ENEIDE DI VIRGILIO
}

\author{
SILVIA TANTIMONACO \\ Universitat de Barcelona \\ silvia.tantimonaco@gmail.com
}

\begin{abstract}
RIASSUNTO
In questo articolo, presentiamo uno studio dettagliato delle attestazioni della parola "Manes" contenute nell'Eneide, analizzandone gli usi e i significati specifici. Li confrontiamo quindi con le fonti letterarie di epoca repubblicana, prestando particolare attenzione alle innovazioni semantiche operate da Virgilio. Infine, contestualizziamo il culto degli dei Mani nell'epoca augustea mediante il ricorso alla documentazione epigrafica.
\end{abstract}

PAROLE CHIAVE: Manes, Virgilio, Eneide, età augustea, epigrafia latina, formula Dis Manibus.

\section{STANT MANIBUS ARAE. THE MANES IN VERGIL'S AENEID}

\section{ABSTRACT}

In this paper, we offer a detailed analysis of the use of the word "Manes" in the Aeneid. We make a comparison between the different meanings of the word in the previous republican sources, with particular focus on the conceptual innovations introduced by Vergil. We also refer to some epigraphical documents, in order to contextualize the cult of the Manes in the age of Augustus.

KEYWORDS: Manes, Virgil, Aeneid, the Age of Augustus, Latin epigraphy, Dis Manibus formula.

\section{INTRODUZIONE}

I Manes, esseri vincolati alla sfera del post mortem romano, sono definiti dalle fonti antiche e dai loro commentatori con una certa mutevolezza. Inoltre, vengono talora confusi con altri esseri dal carattere sovrannaturale, quali le Larvae, i Lemures, i Lares, i Penates, etc. Per altra parte, è opinione comunemente accettata che il termine Manes abbia subito, nel corso del tempo, un processo evolutivo e un ampliamento dei suoi referenti semantici. ${ }^{1}$

Un fenomeno di particolare interesse si dà nell'ambito dell'epigrafia funeraria, dove i Mani cominciano ad essere menzionati soltanto a partire dall'età di Augusto, specialmente, com'è noto, nella formula Dis Manibus (sacrum), destinata a diffondersi in maniera capillare ed estensiva in Italia e in tutte le province dell'Impero. ${ }^{2}$ Al di fuori di tale contesto, i Manes sono testimoniati in epigrafia specialmente nei $C L E$, dove ricorrono soprattutto all'interno di eco letterarie, $\mathrm{e}$,

1 Per i Manes e gli altri esseri che popolano la sfera del post mortem romano e la relativa bibliografia, rimandiamo a Tantimonaco (2015: 109, nt. 2 e 3).

2 In generale su questa formula, cf. Herman (1998). 
più di rado, in iscrizioni non metriche, le più antiche delle quali si datano comunque nella prima età augustea. ${ }^{3}$

La constatazione di un termine post quem così specifico ci ha indotti a studiare gli usi e i significati della parola Manes all'interno della produzione virgiliana, in particolare nell'Eneide ${ }^{4}$. Quest'opera, infatti, compendiò l'intera tradizione romana anteriore a sé e, allo stesso tempo, seppe esprimere pienamente l'epoca del princeps e le sue novità; inoltre, trovò un'amplissima diffusione, come dimostrano i non rari graffiti che, un po' in tutto l'Impero, riproducono a memoria e in maniera del tutto spontanea alcuni passi del medesimo poema. ${ }^{5}$

\section{II. "MANES" PRIMA DI VIRGILIO}

Nelle fonti letterarie di epoca anteriore a Virgilio, i Manes sono menzionati con scarsa frequenza e con un significato quasi esclusivo che designa il gruppo collettivo dei defunti, in contrapposizione agli dei Superi e agli uomini. ${ }^{6}$

Una fonte antica identificabile con Ateio o Catone, citata da Festo, colloca gli Dei Manes all'interno del mundus, la fossa rituale di fondazione della città, a loro consacrata, che si apriva tre giorni all'anno: 24 agosto, 5 ottobre e 8 novembre, date in cui era imposta la cessazione di tutte le attività umane. ${ }^{7}$ La connessione dei Mani con il mondo infero risulta quindi evidente per la loro ubicazione nella cavità della terra; inoltre, se ne sottolinea il carattere religioso:

FEST. p. 157: Eius (scil. mundi) inferiorem partem veluti consecratam Dis Manibus clausam omni tempore, nisi his diebus qui supra scripti sunt, maiores $c . . m$; quos dies etiam religiosos iudicaverunt ea de causa, quod quo tempore ea, quae occultae et abditae religionis Deorum Manium essent, veluti in lucem quandam adducerentur et patefierent, nihil eo tempore in republica geri

\footnotetext{
${ }^{3}$ I più antichi epitaffi sinora noti a Roma contenenti la formula di dedica ai Mani sono: CIL I 2, 761 (= XIV 2464); 1273 (= VI 14574). Inoltre, tra gli esempi più antichi: CIL VI 2489 (29 d.C.); 22668 (33 d.C.) e 7303 (58 d.C.), cf. Solin (1971: 36, nt. 1). Tra le iscrizioni più antiche in cui troviamo menzione dei Mani fuori dalla formula di adprecatio abbiamo invece: CIL VI 37528, dell'ultimo quarto del I sec. a.C. / primo quarto del I d.C. (cf. EDR029224 del 07-12-2014) e 1527, datata tra l'8 e il 2 a.C. (cf. Durry 1950: LIV).

${ }^{4}$ Ci serviremo della seguente edizione: J. Perret, Virgile. Énéide, I-III, «Les Belles Lettres », Paris, $1981^{2}$.

${ }^{5} \mathrm{Si}$ pensi soltanto alle numerosissime volte in cui l'incipit del poema è ripetuto nei graffiti di Pompei, anche con variazioni estrose, come nel caso di CIL IV 9131 (Fullones ululamque cano non arma virumq(ue)). Per il caso della Hispania, cf. Mayer 1993. Ma l'estensione delle citazioni coinvolge davvero i quattro punti cardinali dell'Impero, come dimostrano per esempio: IILPecs 296 (Pannonia Superior), Vindolanda 452 (p 3, 160) (Britannia), MonsClaudianus-OGL-01, 190 (Aegyptus) e CIL II 4967, 31 (Baetica). Per le abbreviazioni dei corpora citati nel corso di tutta la trattazione, rimandiamo al Database Clauss-Slaby (http://www.manfredclauss.de/abkuerz.html).

${ }^{6}$ Fondamentale per lo studio delle occorrenze della parola Manes in letteratura (oltre che in epigrafia) è la consultazione di Bömer (1936).

${ }^{7}$ Sul tema del mundus, cf. la bibliografia fornita da De Sanctis (2014: 211-212, nt. 40) alla quale aggiungiamo anche Santangeli Valenzani (2014).
} 
voluerunt. Itaque per eos dies non cum hoste manus conserebant: nọ exercitus scribebatur: non comitia habeba<ntur: non> aliud quicquam in republica, nisi quod ultima necessitas admonebat, administrabatur. ${ }^{8}$

Molto importante è quindi il passo del De legibus, in cui Cicerone, citando l'antica legge della Dodici Tavole, fa riferimento, per un lato, alla pratica della divinizzazione dei defunti e, per altro lato, alla sacralità dei diritti degli dei Manes. Ciò è utile a comprendere, almeno in parte, il motivo dell'estensione massiccia della formula epigrafica Dis Manibus sacrum sui sepolcri romani, che avrebbe contribuito a qualificare la tomba come res religiosa, sancendone per tanto l'inviolabilità':

Cic. leg. II 9, 22: Deorum Manium iura sancta sunto. Sos leto datos, divos habento.

Il concetto del rispetto dovuto ai Mani è espresso dallo stesso autore anche nei Topica, dove si opera una distinzione tra dei Superi, Mani e uomini, ai quali corrispondono, rispettivamente, i concetti della pietas, della sanctitas e della iustitia o aequitas:

Cic. top. 90, 7-10: Atque etiam aequitas tripertita dicitur esse: una ad superos deos, altera ad manes, tertia ad homines pertinere. Prima pietas, secunda sanctitas, tertia iustitia aut aequitas nominatur.

È quindi conveniente ricordare che una simile gerarchia tripartita si trova già negli autori greci. Pindaro, ad esempio, distingue tra dei, eroi e uomini; ${ }^{10}$ per Platone, invece, le categorie sono quattro, cioè: dei, eroi, demoni e uomini, però con la possibilità che il termine daimon sia usato come alternativa per heros, ad indicare una condizione di esistenza interstiziale tra uomini e dei. ${ }^{11} \mathrm{Nel}$ pensiero greco, infatti, eroi e demoni assumono tendenzialmente un carattere intermedio e sono dotati di poteri sovrannaturali, essendo anche destinatari di un culto. ${ }^{12}$ In un passo delle verrine, i Manes sono rappresentati come gli spiriti dei defunti, in grado di sollevare le Pene e le Furie per provocare vendetta nel mondo umano:

Cic. Verr. II, 5, 113: Non testium modo catervas, cum tua res ageretur, sed ab dis Manibus innocentium Poenas sceleratorumque Furias in tuum iudicium esse venturas.

\footnotetext{
${ }^{8}$ L'autore continua spiegando l'etimologia di manalis e di Manes: Manalis fons appellatur ab auguribus puteus perennis, neque tamen spiciendus videtur, quia flumen id spiciatur, quod sua sponte in amnem influat. Manes di ab auguribus invocantur, quod $i$ per omnia aetheria terrenaque ma<nare credantur. Idem di su>peri atque inferi <dicebantur> . . . Nonostante ciò, è forse più plausibile l'etimologia fornita da Varrone ed altri autori, per la quale cf. infra, in corpo di testo.

${ }^{9}$ Cf. De Filippis Cappai (1997: 113 e 118).

${ }^{10}$ Cf. Pi. O. II. La stessa tripartizione in Antifonte (I, 27).

${ }^{11}$ Cf. Pl., Cra., 397e-399c. Vedasi Böhringer (2001: 31-32).

12 Vedansi Dillon (2000: in particolare le pp. 103-106 e 109-116).
} 
Il carattere potenzialmente maligno dei Mani è evidenziato anche nella In Pisonem, lì dove si fa riferimento alla necessità di un piaculum per placare i Manes dei congiurati di Catilina, già giustiziati:

Cic. Pis. 16: A me quidem etiam poenas expetistis quibus coniuratorum Manis mortuorum expiaretis.

In quest'ultimo caso, quindi, il concetto di Manes pare avvicinarsi a quello degli spiriti irrequieti e vendicativi che, generalmente, è designato con il termine Lemures. $^{13}$ Inoltre, ci troviamo dinnanzi ad un primo esempio di "personalizzazione" dei Mani, seppur ancora ristretta ad un gruppo limitato di individui.

Un'accezione simile della parola ricorre in Varrone, quando riferisce di una cerimonia pubblica finalizzata a pacificare i Mani degli schiavi. Se pensiamo alle condizioni vitali di buona parte di questa categoria sociale, ai possibili maltrattamenti subiti, e, soprattutto, al fatto che, come ci dice lo stesso Varrone, i cadaveri degli schiavi non ricevevano per forza sepoltura, ma venivano gettati senza pietà all'interno di fosse comuni (puticoli), possiamo capire la necessità di realizzare periodicamente un piaculum, come forma di protezione preventiva dalla vendetta di tali anime inquiete e risentite:

VARR. ling. VI, 24: Hoc sacrificium fit in Velabro, qu[i]a in novam viam exitur, ut aiunt quidam ad sepulcrum Accae, ut quod ibi; prope faciunt diis Manibus servilibus sacerdotes; qui uterque locus extra urbem antiquam fuit non longe a porta Romanula, de qua in priore libro dixi.

È quindi nuovamente Varrone a fornirci la prima etimologia del termine Manes, che sarebbe derivato dal latino arcaico "bonus" (VARR. ling. VI, 4: Bonum Antiqui dicebant manus). Tale origine è confermata da Macrobio e da Festo, ed è citata da Servio, che ne sottolinea l'uso antifrastico (katà antífrasin), avente il fine di scongiurare la tendenza malevola di questi esseri infernali ${ }^{14}$.

Da ultimo, l'identificazione dei Mani con i defunti è confermata da Sant'Agostino, il quale, citando ancora Varrone, riferisce contrariato che i Romani avevano l'abitudine di divinizzare i morti:

AUG. civ. 8, 26: Varro dicit omnes ab eis mortuos existimari Manes deos.

D'altra parte, la menzione dei Mani insieme alle divinità infernali all'interno dell'antico carmen devozionale, con il quale il generale romano votava se stesso e il proprio esercito all'Ade, riconferma senz'ombra di dubbio la connessione dei

${ }^{13}$ Cf. Hild (1904: 1100-1101).

${ }_{14}$ Cf. MACR. Sat. 1, 3, 13; FEST. p. 132 L; SERV. Aen. 1, 139; 3, 63. 
Mani con gli Inferi e con le potenze ctonie. ${ }^{15}$ Il radicamento dei Mani nelle credenze popolari è invece confermato da Lucrezio, nella cui opera vi si allude come ai destinatari dei riti funebri e anche come ai traghettatori delle anime all'Orco ${ }^{16}$.

Vediamo quindi che, nelle fonti di epoca repubblicana, la parola Manes è impiegata per designare, in generale, il collettivo indistinto dei defunti o anche le anime dei trapassati nel loro insieme. Tale gruppo di anime si restringe talora, come nel caso dei Mani dei congiurati o degli schiavi, attestati, rispettivamente, in Cicerone e Varrone, uso in cui, però, la parola si configura quasi come un sinonimo di Lemures.

Inoltre, si delinea in origine una visione tendenzialmente negativa dei Manes, basata sulla credenza che fossero in grado di provocare fenomeni sovrannaturali, servendosi dei loro poteri semidivini e ricorrendo alla loro connessione con gli dei inferi. Per tale motivo, i Mani erano oggetto di rispetto e di cerimonie periodiche, finalizzate a mantenere la pax e la conseguente serenità nel mondo dei vivi.

\section{III. "MANES" IN VIRGILIO}

\section{Occorrenze e aspetti formali del termine "Manes"}

Virgilio è uno degli autori che più abbondantemente fa uso del sostantivo "Manes" ed è sicuramente il primo a impiegarlo in maniera così massiccia. La parola compare quattro volte nei Georgica, la cui composizione si situa tra il 36 e il 29 a.C. Qui, assume sempre il significato di divinità infernali oppure di Oltretomba. ${ }^{17}$

Nell'Eneide, invece, la parola è attestata ben 22 volte, e, come prevedibile, ricorre con frequenza maggiore nei libri la cui tematica è in vario modo collegata all'avvenimento di morti o alla menzione di defunti o di forze infernali, come dimostra la tabella riportata a continuazione:

\begin{tabular}{ccc}
\hline Libro & $\begin{array}{l}\text { Nr. } \\
\text { sostantivo Manes }\end{array}$ & $\begin{array}{c}\text { occorrenze } \\
\text { del }\end{array}$ \\
\hline I & 0 \\
\hline II & 0
\end{tabular}

\footnotetext{
${ }^{15}$ Cf. MACR. Sat. 3, 9.

${ }^{16}$ Cf. LUCR. nat. III, 52; VI, 761-764.

${ }^{17} \mathrm{Si}$ tratta dei seguenti passi: VERG. georg. 1, 243 (sub pedibus Styx atra videt Manesque profundi); 4, 469 (ingressus, Manisque adiit regemque tremendum); 4, 489 (ignoscenda quidem, scirent si ignoscere Manes); 4, 505 (Quo fletu Manis, quae numina voce moveret?).
} 


\begin{tabular}{cc}
\hline III & 3 \\
\hline IV & 4 \\
\hline V & 1 \\
\hline VI & 4 \\
\hline VII & 0 \\
\hline VIII & 1 \\
\hline IX & 0 \\
\hline$X$ & 6 \\
\hline$X I$ & 1 \\
\hline$X I I$ & 2 \\
\hline
\end{tabular}

Si contraddistinguono, infatti: il libro III, che contiene il nostos di Enea e i suoi compagni da Troia e la narrazione dei pericoli ad esso correlati; il IV, incentrato sull'amor/furor di Didone, la quale, abbandonata da Enea, medita vendetta mediante il ricorso alla magia nera; il VI, dove trova spazio la katabasis; e il X, in cui vengono descritti gli scontri bellici tra Troiani e Latini per la supremazia del potere nel Lazio.

Dal punto di vista morfosintattico, la parola, che è un plurale tantum, ${ }^{18}$ compare nelle forme e usi che si elencano di seguito:

\begin{tabular}{ll}
\hline Forma flessa & Funzione sintattica \\
\hline Manes & 3 nom. \\
& 1 acc. \\
& 1 voc. \\
& 15 acc. \\
\hline Manis & 2 dat. \\
\hline
\end{tabular}

L'analisi metrica delle occorrenze della parola Manes nelle sue differenti forme flesse rivela che "Manis" e "Manes" costituiscono ineccepibilmente uno spondeo (- -), ${ }^{19}$ il quale, in tre casi soltanto, contribuisce a risolvere in maniera funzionale la chiusura dell'esametro, mentre più spesso ricorre in associazione

\footnotetext{
${ }^{18}$ Ce lo confermano i grammatici antichi, cf. Tantimonaco (2015: 111-112, nt. 14, con indicazione dei passi specifici).

${ }^{19}$ Cf. Quicherats (1852: 650).
} 
con particelle enclitiche (Manisque / Manesve / Manisve) per creare la fine di uno spondeo e l'inizio di un dattilo, spesso con cesura intercalata (- I - `). Pertanto, la scelta dell'una o dell'altra variante da parte del poeta sembra essere del tutto dipendente da motivi stilistici e ritmici, in particolare nel caso dell'accusativo, che presenta entrambe le varianti, tra le quali è privilegiata l'opzione "Manis". ${ }^{20} \mathrm{Dal}$ punto di vista stilistico, il termine è sovente associato all'aggettivo imus, con il quale compare quasi sempre in apallage, creando un gioco ritmico efficace e suggestivo (Manis... sub imos; Manisque... ad imos).

\section{Significati della parola Manes}

Elenchiamo di seguito i differenti significati acquisiti dalla parola Manes all'interno dell'Eneide, citando caso per caso i passi testuali di riferimento. Nella prima sezione, prendiamo in analisi i casi in cui Manes possiede dei referenti reali concreti, che non presuppongono alcuna innovazione importante rispetto agli usi della parola già riscontrati nelle fonti anteriori (cf. supra, par. II); di seguito, includiamo i referenti metaforici da essi direttamente derivati.

\subsection{Referenti concreti:}

- Gli dei inferi: vengono esplicitamente contrapposti ai Superi, sebbene non siano mai menzionati insieme all'epiteto dei. Al pari degli dei Celesti, i Mani hanno la capacità d'intervenire sulla sorte degli uomini ed anche di fornire loro responsi:

VERG. Aen. X, 31-35:

Si sine pace tua atque invito numine Troes

Italiam petire, luant peccata neque illos

iuveris auxilio; sin tot responsa secuti

quae Superi Manesque dabant, cur nunc tua quisquam

vertere iussa potest aut cur nova condere fata?

VERG. Aen. X, 39-41:

Nunc etiam Manis (haec intemptata manebat sors rerum) movet et superis immissa repente

Allecto medias Italum bacchata per urbis.

VERG. Aen. XII, 645-647:

Terga dabo et Turnum fugientem haec terra videbit?

${ }^{20}$ Entrambe le forme sono accettate dai grammatici, cf. DIOM. gramm. I, 25-30: Nomina quae ablativo casu numero singulari i littera finiuntur genere masculino et feminino sublata $i$ ultima et apposita es syllaba producta faciunt nominativum et vocativum plurales, ut ab hoc et hac agili hi et hae agiles et o agiles. Nam accusativus iuxta regulam manente i littera debet enuntiari, hos et has agilis, ut est "omnis homines"; sed eum ad nominativi et vocativi formam consuetudo transduxit. Cf. anche GELL. XIII, 21, 3: Et Probum ait respondisse: Quo suam Vergilius percontatus est, qui diversis in locis "urbis" et "urbes" dixit arbitrio consilioque usus auris. 
Usque adeone mori miserum est? Vos, o mihi, Manes,

este boni, quoniam Superi aversa voluntas.

- Il luogo a cui si recano e dove si trovano i defunti: in questo senso, la parola compare in espressioni del tipo "concedere ad Manis" o "descendere ad Manes" per indicare il processo del morire (cf. infra). Indicando l'Oltretomba, il termine si associa talora all'aggettivo imus e alla preposizione sub, che sottolineano la profondità del luogo e la sua ubicazione sotterranea:

VERG. Aen. IV, 384-388:

[...] Sequar atris ignibus absens

et, cum frigida mors anima seduxerit artus,

omnibus umbra locis adero. Debis, improbe, poenas.

Audiam et haec Manis veniet mihi fama sub imos.

VERG. Aen. X, 817-820:

Transiit et parmam mucro, levia arma minacis, et tunicam, molli mater quam neverat auro, implevitque sinum sanguis; tum vita per auras concessit maesta ad Manis corpusque reliquit.

VERG. Aen. XI, 179-181:

[...] Meritis vacat hic tibi solus

fortunaeque locus; non vitae gaudia quaero, nec fas, sed gnato Manis perferre sub imos.

VERG. Aen. XII, 648-649:

Sancta ad vos anima atque istius inscia culpae descendam, magnorum haud umquam indignus avorum.

VERG. Aen. XII, 883-884:

[...] O quae satis ima dehiscat terra mihi Manisque deam demittat ad imos?

- Gli abitanti dell'Oltretomba: cioè la collettività delle anime, residenti nell'inframondo oscuro, che tremano alla sola apparizione della luce. Questo significato compare soltanto in un passo del poema, sebbene l'associazione dei Mani con il mondo delle tenebre sia ricorrente, in base al topos della contrapposizione tra vita/luce/giorno e morte/oscurità/notte (cf. punto seguente):21

VERG. Aen. VIII, 241-246:

At specus et Caci detecta apparuit ingens

regia et umbrosae penitus patuere cavernae,

non secus ac si qua penitus vi terra dehiscens

${ }^{21}$ Si pensi soltanto ai celebri versi di Catullo: Soles occidere et redire possunt: / nobis, cum semel occidit brevis lux, / nox est perpetua una dormienda (CATVLL. 5, 4-6). 
infernas reseret sedes et regna recludat

pallida, dis invisa, superque immane barathrum

cernatur, trepident immisso lumine Manes.

- Degli esseri associati con incantesimi ed inganni della mente, compresi i sogni notturni: ${ }^{22}$

VERG. Aen. IV, 487-491:

Haec se carminibus promittit solvere mentes quas velit, ast aliis duras immittere curas, sistere aquam fluviis et vertere sidera retro, nocturnosque movet Manis ${ }^{23}$ : mugire videbis sub pedibus terram et descendere montibus ornos.

VERG. Aen. VI, 893-896:

Sunt geminae Somni portae, quarum altera fertur

Cornea, qua veris facilis datur exitus umbris,

altera candenti perfecta nitens elephanto,

sed falsa ad caelum mittunt insomnia Manes.

2.2. Referenti metaforici derivati per estensione dai significati precedenti:

- Il destino personale dopo la morte: questo riferimento è contenuto in un solo passo, già celebre e dibattuto, in cui la parola Manes, unita al verbo patior e all'idea di individualità (quisque... suos), sembra corrispondere ad una legge del contrappasso, da cui dipende la qualità del destino dopo la morte. ${ }^{24}$ In tal caso, Manes corrisponderebbe all'idea greca di daimon ${ }^{25}$ così come già riscontrata nella letteratura greca arcaica e poi nella tragedia classica, dove la parola assume il significato di "destino, fortuna":26

VERG. Aen. VI, 739-747:

Ergo exercentur poenis veterumque malorum supplicia expedunt: aliae panduntur inanes suspensae ad ventos, aliis sub gurgite vasto infectum eluitur scelus aut exuritur igni; quisque suos patimur Manis. Exinde per amplum mittimur Elysium et pauci laeta arva tenemus donec longa dies perfecto temporis orbe

${ }^{22}$ Così è anche per Tibullo: Ne tibi neglecti mittant mala somnia Manes (TIB. eleg. II, 6, 37).

${ }^{23}$ L'espressione movere Manes si ritrova anche nel verso X, 39 (cf. supra).

${ }^{24}$ Questa è l'interpretazione che, per esempio, dà del verso in questione Thomas (1987); si veda, inoltre, Setaioli (1967).

${ }^{25}$ Cicerone, in realtà, crede che i daimones greci siano più simili ai Lares: Quos Graeci daimonas appellant, nostri, opinor, Lares, si modo hoc recte conversum (CIC. Tim. 38, 1). Sulla nozione di daimon nelle fonti letterarie greche, si veda l'approfondito contributo di Suárez de la Torre (2000).

${ }^{26}$ Cf. Suárez de la Torre (2000: 65-68). Si consideri poi l'esistenza, in greco, di espressioni idiomatiche quali "katà daimona", cf. Hdt. 1, 111. 
concretam exemit labem purumque relinquit

aetherium sensum atque aurai simplicis ignem.

- I resti mortali del defunto, in associazione alle ceneri del cadavere: in questo caso, i Manes sono privi di sensazioni fisiche e di qualità morali, che in altri contesti, invece, possiedono, quando compaiono in qualità di spiriti o divinità dei defunti. In quanto spoglie, inoltre, se ne ribadisce il carattere sacro e inviolabile:

\author{
VERG. Aen. IV, 31-34: \\ Anna refert: "O luce magis dilecta sorori, \\ solane perpetua maerens carpere iuventa \\ nec dulcis natos Veneris nec praemia noris? \\ Id cinerem aut Manis credis curare sepultos?» \\ VERG. Aen. IV, 425-427: \\ Non ego cum Danais Troianam exscindere gentem \\ Aulide iuravi classemve ad Pergama misi, \\ nec patris Anchisae cineres Manisve revelli.
}

- Un luogo profondo: si tratta di un uso metaforico "estremo" che appare solamente in un verso, nel contesto di una tempesta marina, quando Enea e i suoi compagni vengono scaraventati negli abissi da un'onda impetuosa. Ancora una volta, per tanto, si conferma l'ubicazione dei Mani nelle viscere della terra insieme agli dei inferi, nonché la contrapposizione di questi con gli dei celesti, per mezzo dell'immagine della nave che viene spinta, prima, in alto verso il cielo e, dopo, così in fondo da raggiungere "i Mani profondi":

VERG. Aen. III, 564-565:

Tollimur in caelum curvato gurgite, et idem

subducta ad Manis imos desedimus unda.

Alcune osservazioni di carattere generale s'impongono, dunque, in seguito a questa prima rassegna dei significati assunti dal termine Manes nell'Eneide:

I. La principale innovazione operata da Virgilio, rispetto alle fonti letterarie anteriori, è rappresentata dall'ampliamento semantico dei significati basici di Dei Inferi e di comunità dei defunti, per designare anche: il luogo in cui gli dei ctoni e i trapassati risiedono; il destino dell'individuo dopo la morte; i resti mortali del defunto e, infine, un luogo profondo in generale;

II. I Manes vengono relazionati da Virgilio con l'idea di oscurità, notte, tenebra e altri campi semantici affini, che tradizionalmente si riferiscono al fenomeno della morte, contrapposto alla vita, alla quale invece si allude tramite l'idea di luce, chiarità e sole. Si mantiene, per tanto, la connotazione tendenzialmente negativa già riscontrabile negli autori anteriori; 
III. Tutti i significati di Manes presenti nell'Eneide trovano riscontro in epigrafia: ${ }^{27}$ in alcune varianti locali della formula di consacrazione del sepolcro, incontriamo la dicitura Dis Inferis Manibus;"28 l'associazione dei Manes con l'Oltretomba è invece suggerita dalla loro rappresentazione, non di rado, come agenti della morte, che rapiscono o più semplicemente accolgono i nuovi defunti nel proprio novero; ${ }^{29}$ in altri casi, invece, essi sono evocati per i loro poteri sovrannaturali e magici; ${ }^{30}$ non sono poi pochi gli esempi in cui la parola indica le spoglie mortali (ossa o ceneri) del defunto; ${ }^{31}$ infine, l'idea del destino individuale dopo la morte sembra identificarsi con i Mani personali, per lo meno nella famosa iscrizione conosciuta come laudatio Turiae, in cui compare la frase augurale: te di Manes tui ut quietam pat[ia]ntur atque ita tueantur opto (anche lì, come si vede, in associazione con il verbo patior $)^{32}$.

\section{Innovazioni concettuali}

Due sono, però, le principali novità di cui la parola Manes fa mostra nell'Eneide di Virgilio: il restringimento di tale concetto per indicare, non solo i defunti nel loro congiunto, ma anche il defunto del singolo, nella sua individualità; e l'uso specifico della parola all'interno della descrizione del culto eroico. Tale evoluzione concettuale presuppone un nuovo modo di impiegare il sostantivo Manes, in associazione con il nome personale del defunto o nell' ambito degli onori funebri.

3.1. La individualizzazione dell'idea di Manes avviene per mezzo dell'associazione con il nome del defunto o con un ristretto gruppo di defunti, come dimostrano i passi elencati a continuazione:

(1) VERG. Aen. IV, 425-427 (già citato supra, par. 2.2):

Non ego cum Danais Troianam exscindere gentem

Aulide iuravi classemve ad Pergama misi,

nec patris Anchisae cineres Manisve revelli.

(2) VERG. Aen. VI, 119-123:

Si potuit Manes accersere coniugis Orpheus

\footnotetext{
${ }^{27}$ Sul tema della presenza di Virgilio nelle iscrizioni metriche, vedasi Hoogma (1959).

${ }^{28}$ Vedasi, a titolo d'esempio: CIL II 238; 2464; III 704; 3378; V 6663; VI 9071, 10147; 12311; X 2322; XIII 530 e molti altri.

${ }^{29}$ Cf. per esempio: CIL VI 6986 = CLE 1034 (me potius Manes rapuissent Ditis avari).

${ }^{30}$ Cf. per esempio: CIL VI 27365 (tu qui legis et / dubitas Manes / esse sponsione / facta invoca / nos et intelleges).

${ }^{31}$ Cf. per esempio: CIL II $2 / 7,569$ (= CLEBetica C9 = CLEHisp 26: adoro parcatis / tumulo Manes quod con/tegit alte).

${ }^{32}$ CIL VI $41062=1527($ p 3142, 3444, 3805) = 31670 (Roma), 8-2 a.C., già citata supra, nt. 3.
} 
Threicia fretus cithara fidibusque canoris,

si fratrem Pollux alterna morte redemit

itque reditque viam totiens. Quid Thesea magnum,

quid memorem Alciden? Et mi genus ab Iove summo.

(3) VERG. Aen. X, 524-525:

Per patrios Manis et spes surgentis Iuli

te precor, hanc animam serves gnatoque patrique.

(4) VERG. Aen. X, 531-534:

Argenti atque auri memoras quae multa talenta, gnatis parce tuis. Belli commercia Turnus sustulit ista prior iam tum Pallante perempto. Hoc patris Anchisae Manes, hoc sentit Iulus.

(5) VERG. Aen. X, 825-830 :

Quid tibi nunc, miserande puer, pro laudibus istis, quid pius Aeneas tanta dabit indole dignum?

Arma, quibus laetatus, habe tua; teque parentum

Manibus et cineri, siqua est ea cura, remitto. ${ }^{33}$

Hoc tamen infelix miseram solabere mortem:

Aeneae magni dextra cadis. [...]

Come si può vedere, sono in tal modo menzionati soprattutto i Mani di Anchise: in quattro dei cinque casi attestati, mentre nel passo (2) il riferimento è ai Mani di Euridice. Le espressioni "patris... Manis", "patrios Manis", "patris Manes" e, soprattutto, "parentum Manibus", richiamano alla memoria quegli Dei Parentes o Dei Parentum, attestati sia in epigrafia che in letteratura, che rappresenterebbero una circoscrizione specifica dei Manes riferita agli avi ${ }^{34}$. Nell'Eneide, i Manes paterni sono rivestiti da un'aura di sacralità, che spinge a realizzare scongiuri in loro nome (per patrios Manis... te precor) e a richiamare gli uomini al doveroso rispetto nei loro confronti (siqua est ea cura). In due occasioni, inoltre, i Mani di Anchise sono contrapposti al giovane Iulo, "speranza delle generazioni future" (spes surgentis Iuli).

I Manes così "personalizzati" corrispondono quindi alle anime dei singoli defunti, ${ }^{35}$ sono dotati di un nome proprio e di una identità personale e inoltre, come si deduce dall'esempio (4), sono in grado di provare sensazioni affini a quelle umane (Hoc patris Anchisae Manes, hoc sentit Iulus).

\footnotetext{
${ }^{33}$ Compare in questo verso l'immagine di "restituire" i vivi ai Mani (Manibus remittere), vincolata all'idea, espressa alla fine del libro VI, che le anime di quanti sono destinati a nascere (e a marcare la storia, come ad esempio Cesare) stiano già aspettando nell'Ade (cf. VERG. Aen. VI, 756ss.).

34 Per gli Dei Parentes rimandiamo a Liou-Gille (1993: 109-110) e a Bettini (2009: 87-126).

${ }^{35}$ La concezione individualistica dei Mani può essere in parte confrontata con quanto appare, in riferimento ai demoni, in Apuleio, il cui referente principale è Platone, cf. Dillon (2000: 109-116).
} 
3.2. La eroizzazione del concetto di Manes avviene invece mediante l'associazione dei Mani con i gesti e i luoghi del culto funerario, secondo un modello diffuso sin dal V sec. a.C. in Grecia e in Magna Grecia: ${ }^{36}$

(6) VERG. Aen. III, 62-68:

Ergo instauramus Polydoro funus et ingens aggeritur tumulo tellus; stant Manibus arae, caeruleis maestate vittis atraque cupresso, et circum Iliades crinem de more solutae; inferimus tepido spumantia cymbia lacte sanguinis et sacri pateras, animamque sepulcro condimus et magna supremum voce ciemus.

(7) VERG. Aen. III, 300-305:

Progredior portu classis et litora liquens, sollemnis cum forte dapes et tristia dona ante urbem in luco falsi Simoentis ad undam libabat cineri Andromache Manisque vocabat Hectoreum ad tumulum, viridi quem caespite inanem et geminas, causam lacrimis, sacraverat aras.

(8) VERG. Aen. VI, 500-508:

Deiphobe armipotens, genus alto a sanguine Teucri, quis tam crudelis optavit sumere poenas? Cui tantum de te licuit? Mihi fama suprema nocte tulit fessum vasta te caede Pelasgum procubuisse super confusae stragis acervom. Tunc egomet tumulum Rhoeteo in litore inanem constitui et magna Manis ter voce vocavi. Nomen et arma locum servant; te, amice, nequivi conspicere et patria decedens ponere terra.

(9) VERG. Aen. V, 94-99:

Hoc magis inceptos genitori instaurat honores, incertus geniumve loci famulumne parentis esse putet; caedit binas de more bidentis totque sues, totidem nigrantis terga iuvencos, vinaque fundebat pateris, animamque vocabat Anchisae magni Manisque Acheronte remissos.

36 Il culto degli eroi, così come lo troviamo in Virgilio, non è ancora presente nel modello omerico, dove la parola "heroes" designa genericamente i guerrieri di nobile lignaggio, molti dei quali diverranno, a partire dal siglo V a.C., oggetto di venerazione in Grecia, specialmente a seguito della costruzione di cenotafi in loro memoria, cf. Böhringer (2001: 30). Lo stesso Enea, comunque, possedeva un heroon a Lavinium, per il quale vedasi in particolare Fulminante Brocato (2000), con bibliografia anteriore (cf. anche: Liou-Gille, 92-99; Schilling 1980, 141-144 e 151-152). Sugli eroi greci, rimandiamo alla lettura di Brelich (1958). Sull'influenza e le differenze tra la tradizione greca e quella latina, vedasi Schilling (1980: specialmente 146-152). 
Nel caso di Polidoro (6), la celebrazione dei rituali funebri equivale ad un piaculum per la morte violenta e senza sepoltura subita dal guerriero, del quale Enea incontra casualmente l'anima tormentata. Ettore (7), invece, viene onorato a distanza dalla sua antica sposa, Andromaca, che ora risiede a Butroto, insieme al nuovo marito, Eleno. Ai già menzionati Mani di Anchise (8) viene reso onore in occasione del primo anniversario della morte, nel celebre episodio del libro $\mathrm{V}$ in cui si verifica il prodigium dell'apparizione del serpente che fuoriesce dalla tomba (vv. 85-93) ed hanno luogo i ludi funebri. ${ }^{37}$ Infine, i Mani di Deifobo sono evocati a gran voce in corrispondenza del suo cenotafio in terra retea (9). A questo proposito, è importante evidenziare la presenza, in tutti gli episodi citati, dell'elemento del tumulus inanis, come tipico del culto eroico dei guerrieri greci. Inoltre, sia nel primo che nel secondo gruppo di passi (1-5 e 6-9), il ricorrere frequente di vocare e di altri verbi relazionati con la sfera semantica del chiamare (come accersere) in connessione con il culto dei Mani, soprattutto dinnanzi alle tombe o ai cenotafi dei defunti, sembra indicare che la comunicazione diretta dei vivi con i trapassati non è più possibile. Essa, cioè, deve passare necessariamente attraverso i Mani, perché il defunto è già divenuto "i suoi Mani" ed è unicamente con questi che i vivi possono e devono ormai interagire, rievocandoli dalla loro ubicazione inframondada (cf. infra). Inoltre, va notato che il verso: "et magna Manes ter voce vocavi", riportato nel passo (9) contiene, dal punto di vista formale, un perfetto esempio di Lautmalehrei, e, dal punto di vista culturale, una riassuntiva descrizione del rito del funerale, che prevedeva l'invocazione, a gran voce, del nome del defunto, presente per altro anche nell'esempio (6)..$^{38}$

3.3. Se, come abbiamo detto, tutti i significati assunti dalla parola Manes che abbiamo visto nella sezione precedente si ritrovano anche nella documentazione epigrafica, specialmente nei $C L E$, riteniamo invece che questi ultimi due usi della parola, nel significato di anima individuale del defunto e di eroe, si concentrino specialmente nella dedica funeraria Dis Manibus, che di solito appare seguita dal nome personale del defunto (per lo più, in caso genitivo o dativo) ${ }^{39} \mathrm{e}$ che entra in uso a partire dall'età augustea. In effetti, attraverso una selezione di documenti epigrafici coevi, vedremo a continuazione come il princeps abbia promosso il culto dei Mani di alcuni esponenti della sua famiglia imperiale, presentati e avvertiti a livello popolare come dei veri e propri eroi, e come questo abbia avuto delle ripercussioni tra la gente comune, secondo una pratica di imitatio.

\footnotetext{
${ }^{37} \mathrm{Su}$ questo episodio, cf. in particolare Bayet (1971), che ricorda anche come il serpente, oltre a comparire spesso nelle rappresentazioni funerarie, sia associato alla forma assunta da taluni eroi greci, quali Cecrope o Erittone, nel mito, oppure alle parvenze di dei ed eroi guerrieri nelle narrazioni oniriche (ibid: 375$)$.

${ }^{38}$ Si tratta della pratica rituale della conclamatio, per la quale cf. De Filippis Cappai (1997: 51).

${ }^{39}$ Cf. Herman (1998).
} 


\section{Augusto E I MANES}

Che il culto dei Manes si sia diffuso in epoca augustea a partire da un'iniziativa promossa (e imitata) dall'alto sembra essere comprovato da alcuni documenti epigrafici di tenore ufficiale, che prescrivono la commemorazione dei defunti della casa imperiale.

Il primo indizio, in ordine cronologico, è costituito dai cosiddetti Decreta Pisana, due provvedimenti con cui, su imitazione di analoghe misure assunte dal Senato romano, l'ordo pisanus introduceva e regolamentava nel calendario della colonia le cerimonie da svolgersi, sia a livello pubblico che privato, nel giorno della ricorrenza della morte di Lucio Cesare e di suo fratello Caio. ${ }^{40}$ I due principes iuventutis, defunti, l'uno, nel 2 d.C. a Marsiglia e, l'altro, nel 4 d.C. in Licia, erano stati sepolti a Roma, nel mausoleo di Augusto; pertanto, la realizzazione delle inferiae per i loro Mani (diis Manibus eius) nella città provinciale di Pisa, doveva avvenire in corrispondenza di cenotafi, e dinnanzi alle are per essi predisposte:

[...] utique / apud eam aram quodannis a(nte) d(iem) X[II K(alendas) Sept(embres) p]ublice Manibus eius per magis/tratus eosve, qui ibi iuri dicendo pr[ae]runt, togis pullis amictos, I quibus eorum ius fasque erit eo die [eiu]s vestis habendae, inferiae mit/tantur, bosque et ovis atri infulis caerulis infulati diis Manibus eiu[s] / mactentur eaeque hostiae eo loco adoleantur superque eas I singulae urnae lactis mellis olei fundantur, ac tum demum fact[am] / c[eteris p]otestatem, si qui privatim velint Manibus eius inferias mitter[e / nive quis] amplius uno cereo unave face coronave mittat $[\ldots] .41$

[...] ut[ique] eo die quodannis publice Manibus eius per magistratus eosve, / q[ui Pi]sis iure dicundo praerunt, eodem loco eodemque modo, quo / L(ucio) C[aes]ari parentari institutum est, parentetur $[\ldots] \cdot{ }^{42}$

È evidente che questi due provvedimenti continuano l'operazione di promozione del culto imperiale, già preannunciata, tra le altre cose, dalla proclamazione del divus Iulius, che faceva di Augusto il divi filius. ${ }^{43}$ Nonostante ciò, sussiste una differenza sostanziale tra il processo di divinizzazione del principe -che, iniziata già in vita, si sarebbe completata soltanto dopo la sua

40 I testi dei due provvedimenti sono riprodotti, rispettivamente, in: CIL XI 1420 e 1421. Rimandiamo inoltre alle edizioni di Sherk (1970: 42-45) e Segenni (2011). Si vedano, poi, gli studi specifici di Scheid (2000) e Segenni (2007).

${ }^{41}$ CIL XI 1420, 11. 15-24

42 CIL XI 1421, 11. 31-33.

43 Sul tema della "stratégie du deuil" impiegata da Augusto per aumentare la visibilità della sua familia e preparare la propria divinizzazione, cf. Hurlet (2013: bibliografia anteriore a p. 63, nt. 3). Recentemente, González ha ribadito l'ipotesi che una vera e propria politica di apotheosis di Augusto fosse stata promossa già in vita dallo stesso princeps, favorendo l'immagine di persona superiore ai comuni mortali e, per tanto, assimilabile alle divinità, cf. González Fernández (2015). La querelle sulla divinizzazione in vita o postuma degli imperatori, comunque, è di lunga tradizione; vedasi, per le due rispettive posizioni: Fishwick (1991) e Clauss (1999). 
morte- e, invece, la eroizzazione di Caio e Lucio Cesare (e, dopo di loro, di altri membri della famiglia imperiale, come Germanico e Druso).

In primo luogo, si tratta di un culto in certo modo indiretto, cioè rivolto non alla persona dei defunti eroizzati, bensì ai loro Mani. Questo fatto rimanda a quanto abbiamo già detto sulla necessità di mediare il culto dei defunti attraverso l'invocazione dei Manes di costoro (cf. supra, par. 3.2); inoltre, a livello pratico, parrebbe spiegare il motivo per cui la dedica degli epitaffi delle persone comuni non è rivolta ad esse direttamente, bensì, di nuovo, ai loro Manes.

Similmente, infatti, all'interno dell'iscrizione conosciuta come Feriale Duranum (cioè ancora nel III sec. d.C.), si registra in corrispondenza del dies natalis di Germanico la celebrazione di una supplicatio in onore della sua memoria, anziché in onore della sua persona. Ciò sembra spiegarsi con il fatto che questi non fu mai divinizzato e, di conseguenza, non poteva essere destinatario di supplicationes. In effetti, già a poca distanza dalla morte di Cesare, nel 44 a.C., Cicerone (Phil. 1, 13) aveva difeso la differenza esistente tra gli onori dovuti ai defunti (nello specifico, le parentationes) e quelli da tributarsi agli dei (cioè, appunto, le supplicationes). ${ }^{4}$ Così, proprio la destinazione di parentationes ai Mani di Caio e Lucio Cesare ne rivelerebbe lo statuto di defunti eroici, uno statuto che, essendo privilegiato rispetto a quello dei comuni mortali defunti, ma, a differenza dei sovrani, soltanto "quasi divino", 45 li collocava a metà strada tra homines e divi, laddove, come abbiamo visto, trovavano tradizionalmente posto gli eroi. ${ }^{46}$

La parentatio prevista per i Mani dei principi, inoltre, non può non richiamare alla memoria, nei modi, quella destinata nell'Eneide ad Anchise (cf. supra). ${ }^{47}$ Virgilio, anzi, sembra operare un'equilibrata fusione tra la tradizione romana relativa agli eroi ecisti, che in realtà ne prevedeva l'ascesa al cielo (fatto che aveva riguardato anche lo stesso Cesare, convertitosi in stella) e la speculazione greca, che invece associava il culto degli eroi con le forze ctonie (alle quali appartengono i Manes) e con l'esistenza di un sepolcro e di un luogo di culto (visto che ad Anchise corrispondono, nell'Eneide, un tumulus ed un lucus). ${ }^{48}$

\footnotetext{
${ }^{44}$ Cf. Fraschetti (2000: 146-147). Sul tema degli "eroi" della familia di Augusto, si veda anche Fraschetti (1984).

${ }^{45}$ Cf. Scheid (2000: 136-137). A proposito della superiorità dei Mani di Anchise rispetto agli altri Mani nel poema di Virgilio, cf. Bayet (1971: 377).

46 Per le differenze tra culto dei defunti, degli eroi e degli dei, rimandiamo a Böhringer (2001: 3746) che, occupandosi nello specifico della realtà greca, individua i principali fattori di distinzione tra i tre tipi di culto nella durata, gruppi e funzione sociale degli stessi.

47 Sulle similitudini tra i riti per Anchise descritti da Virgilio e quelli per i principes contenuti nei documenti pisani, cf. Scheid (2000: 134-136 e 138-139).

${ }^{48}$ Cf. VIRG. Aen. V, 760-761: (...) Tumuloque sacerdos / ac lucus late sacer additur Anchiseo. Cf. soprattutto Schilling (1980: 150-151) e anche Bayet (1971: 381).
} 
A Roma, l'eroizzazione di personaggi eccellenti trova in Romolo, Enea e Servio Tullio i principali antecedenti, ${ }^{49}$ accanto ai quali si ricorderanno anche le parentationes periodicamente tributate ad Acca Larenzia, 50 Tarpeia e Tito Tazio, tutte figure appartenenti all'epoca arcaica e legate alla storia mitica di Roma. Caio e Lucio Cesare costituiscono, pertanto, i primi eroi contemporanei del principato augusteo. ${ }^{51}$

D'altra parte, i provvedimenti per la morte dei giovani principi, elaborati a loro volta su modello romano, ebbero ripercussione su altre località dell'Impero, come dimostra l'esistenza della Tabula Siarensis, un fortunato documento bronzeo procedente dalla città ispana di Siarum, nella provincia della Baetica. ${ }^{52}$ Essa, infatti, contiene un decreto che fu emanato nel 19 d.C. in favore di Germanico, da poco deceduto ad Antiochia, e prevedeva la realizzazione di parentationes in suo onore, con esplicito riferimento alla tipologia di culti già predisposti per Lucio e Caio:

Inferiae dis Manibus eius (...) eodem ritu sacrifici quo / [publice inferiae mitterentur] Manibus C(ai) et L(uci) Caesarum..$^{53}$

Anche in questo caso, il culto dei Mani di Germanico doveva aver luogo, date le circostanze, presso un cenotafio. Nel prescritto si stabilisce, in effetti, che vengano eretti in onore del defunto tre archi funerari dotati di statua e iscrizione (uno a Roma, un altro in Siria e un ulteriore in Germania, presso il Reno, laddove era già stato edificato spontaneamente da parte dell'esercito un tumulo per il padre Druso $)^{54}$ e che, inoltre, gli si costruiscano un tumulus honorarius in Germania ed un sepulchrum ad Antiochia, così come un cenotafio (tribunal) a Epidafna, luogo in cui Germanico era spirato. Ogni anno, in occasione del giorno della morte di Germanico, dovevano compiersi in questi luoghi i già menzionati sacrifici rituali in onore della sua memoria; il giorno del suo compleanno, invece, dovevano tenersi giochi funebri negli anfiteatri di quelle tra le città coinvolte che ne possedessero uno. ${ }^{55}$

Ulteriori documenti di carattere pubblico offrono poi testimonianza del culto dei Mani degli esponenti della casa augustea. Per fare solamente un esempio, a

\footnotetext{
49 Sul culto di questi personaggi, che sono eroi fondazionali, cf. Liou-Gille (1980) e Schilling (1980).

${ }^{50}$ Cf. il passo di Varrone (VARR. ling. VI, 24) cit. supra, par. II.

${ }^{51}$ Cf. Fraschetti (2000: 153-159).

52 CILA II/3, 927. Su questo documento vedasi il contributo di Fraschetti (1988), il quale tratta anche della Tabula Hebana (cf. infra, nt. 55) e inoltre vedasi González - Arce (1988), con edizione completa del testo e traduzione spagnola alle pagine 307-315.

${ }^{53}$ Frag. I, col. a, 11. 4-5.

54 Sul tumulo di Druso in Germania, cf. anche SVET. Claud. 1,3 e DIO. 55, 2, 3.

55 Cf. Tabula Siarensis, frag. I, 11. 9-37, e frag. II, col. a. Provvedimenti analoghi per la commemorazione di Germanico sono ricordati nella cosiddetta Tabula Hebana, dell'anno 20 d.C., per la quale rimandiamo all'edizione con traduzione spagnola di Javier Lomas (1978).
} 
Brixia, nella Regio X, incontriamo la dedica: Dis Manibus / Drusi Caesaris Germ[anici $f(i l i){ }^{56}$ incisa su di un epistilio che si trovava esposto nel foro della città, e che doveva appartenere ad un monumento apposto in onore della gens Iulia.

Possiamo quindi vedere come, accanto alla promozione del culto dei propri Lares e del Genius Augusti, il princeps abbia fomentato anche il culto dei Manes dei suoi illustri familiari, che fu poi assunto e riprodotto in vari luoghi dell'impero, a partire dal modello centrale romano. Le forme e i modi di espressione di questo culto, pur richiamando le parentationes della tradizione romana, rimandano al modello del culto eroico di principi e guerrieri greci, e prevedono, in molti casi, l'esplicita realizzazione di cenotafi ed heroa, come luogo di celebrazione delle ricorrenze annuali.

Pertanto, riteniamo lecito interpretare la diffusione massiva della formula funeraria Dis Manibus -che ricorre per lo più associata al nome personale del defunto- come un riflesso, a livello popolare, delle iniziative promosse da Augusto verso i defunti della propria familia, per favorirne la nascita di un culto di tipo eroico ${ }^{57}$. Ciò spiegherebbe l'assenza di tale formula nelle iscrizioni antecedenti all'età augustea, visto che il più antico documento con tale formula di datazione certa risale proprio al 29 d.C. e corrisponde all'iscrizione di un militare. ${ }^{58}$

Da parte sua, l'opera di Virgilio avrebbe funzionato da catalizzatore per la diffusione dei nuovi modelli di culto funerario e di devozione verso i defunti famigliari, offrendo come esempio di comportamento, come sempre, il pius Aeneas.

\section{CONCLUSIONI}

Dalla disamina delle fonti anteriori a Virgilio, e dal confronto con gli usi della parola Manes presenti nell'Eneide, abbiamo potuto ricavare che, a partire dall'epoca augustea, questo termine consolida la gamma dei propri referenti, sia reali che metaforici, presupponendo in maniera molto più marcata la personificazione del morto a cui "appartengono" i Mani.

L'opera virgiliana appare quindi come la prima e più chiara testimonianza letteraria del trattamento astratto del concetto eroico di Manes, in cui si sommano l'antica credenza romana sui defunti divinizzati e la tradizione greca degli eroi e dei demoni.

La sintesi che ne risulta è rispecchiata anche dalla documentazione epigrafica, come dimostrano i casi di promozione ufficiale, mediante decreto, del culto dei

\footnotetext{
${ }^{56}$ Si tratta di CIL V 4953, sulla quale cf. Gregori (2003: 132).

57 Sull'evoluzione dell'abito epigrafico a partire dall'età augustea e sul peso dei modelli ufficiali nella produzione di iscrizioni a carattere privato, cf. Mayer (2007).

${ }^{58}$ Cf. supra, nt.3.
} 
Mani individuali di alcuni membri della famiglia augustea e, a livello cittadino, da alcuni cenotafi promossi a loro nome e per il loro culto. Tutto ciò si ripropone anche, per imitazione, nell'epigrafia privata, con la realizzazione dei primi epitaffi consacrati agli Dei Mani dei singoli defunti, nella primissima epoca augustea solo a Roma, e poi rapidamente e con enorme impatto su tutto l'Impero. Così, Virgilio, con il suo capolavoro, ci offre ancora una volta del materiale prezioso per leggere ed interpretare sotto vari fronti l'epoca di Augusto e i materiali prodotti in tale epoca.

Per quanto riguarda il culto dei Mani, tuttavia, resta ancora da capire sino a che punto si trattò, almeno in origine, di una promozione ufficiale, esplicitamente vincolata alle riforme religiose operate dal princeps, oppure semplicemente dell'imitazione spontanea di una forma. Quest'ultima opzione ci appare comunque come la più probabile, dato che la propaganda augustea ha dimostrato in varie occasioni di disporre di una forza e di mezzi tali da coinvolgere, in maniera più o meno consapevole e duratura, gli abitanti dell'Impero in tutte le sue fasce sociali e nei quattro angoli del mondo allora conosciuto.

\section{BIBLIOGRAFIA}

BAYET, J. (1971), "Les cendres d'Anchise: dieu, héros, ombre ou serpent? (Virgile, Éneide, V 42-103)", en Croyances et rites dans la Rome antique, 366-381 (= Gedankenschrift für Georg Rohde, Tübingen, 1961, 39-56).

BETTINI, M. (2009), Affari di famiglia: la parentela nella letteratura e nella cultura antica, Bologna.

BRELICH, A. (1958), Gli eroi greci. Un problema storico-religioso, Roma.

BÖHRINGER, D. (2001), Heroenkulte in Griechenland von der geometrischen bis zur klassichen Zeit. Attika, Argolis, Messenien, Berlin.

BÖMER, F. (1936), “Manes” (s.v.), en Thesaurus Linguae Latinae, VIII.1, Lipsiae.

Clauss, M. (1999), Kaiser und Gott. Herrscherkult im römischen Reich, StuttgartLeipzig.

DE FILIPPIS CAPPAI, C. (1997), Imago mortis. L'uomo romano e la morte, Napoli.

DE SACTIS, G. (2014), “In effossa terra. Sacrifici di fondazione, sepolture rituali e vie d'accesso per l'aldilà!", en Studi e Materiali di Storia delle Religioni (Geografie del mondo altro. Prospettive comparative sugli spazi sacri e l'aldilà) 80/1, 198-225.

DiLlon, J (2000), "Seres intermedios en la tradicción platónica tardía", in Seres intermedios. Ángeles, demonios y genios en el mundo mediterráneo, Pérez Jiménez, A., Cruz Andreotti, G. (eds.), Málaga, 89-117.

DurRY, M. (1950), Éloge funèbre d'une matrone romaine (Éloge dit de Turia), Paris.

FISHWICK, D. (1991), The Imperial Cult in the Latin West. Studies in the Ruler Cult of the Western Provinces of the Roman Empire, II/1, Leiden. 
FrAsChetTI, A. (1984), "Morte dei principi ed eroi della famiglia di Augusto", Annali dell'Istituto Universitario Orientale di Napoli. Dipartimento di Studi del Mondo classico e del Mediterraneo antico 6, 151-189.

FrAschetTI, A. (1988), “La Tabula Hebana, la Tabula Siarensis e il iustitium per la morte di Germanico", en Mélanges de l'Ecole française de Rome. Antiquité T. $100, \mathrm{n}^{\circ} 2,867-889$.

FrASCHETTI, A. (2000), "L'eroizzazione di Germanico", en La commemorazione di Germanico nella documentazione epigrafica. Tabula Hebana e Tabula Siarensis (Convegno Internazionale di Studi, Cassino, 21-24 ottobre 1991), Fraschetti, A. (a cura di), Roma, 141-162.

Fulminante, F., BROCATO, P. (2000), “La morte e la divinizzazione di Enea”, en Roma, Romolo, Remo e la fondazione delle città. Roma, Museo Nazionale Romano, Terme di Diocleziano, 28 Giugno - 29 Ottobre 2000, Carandini, A., Cappelli, R. (a cura di), Milano, 213-215.

GONZÁLEZ FERNÁNDEZ, J. (2015), “El culto a Augusto vivo y la devotio popular: el origen del culto imperial", Onoba 3, 15-24.

GonZÁlez, J., ARCE, J. (eds.)(1988), Estudios sobre la Tabula Siarensis, Madrid.

GREGORI, G. (2003), "Integrazioni ed interpretazioni abusive? A proposito di alcune iscrizioni bresciane per i Giulio-Claudi (IIt X V, 86, 638, 736, 1188)*" en Usi e abusi epigrafici. Atti del Colloquio Internazionale di Epigrafia Latina (Genova 20-22 Settembre 2001), Angeli Bertinelli, M.G., Donati, A. (a cura di), Faenza, 113-133.

Herman, J. (1998), “Dis Manibus. Un problème de syntaxe épigraphique”, en Estudios de lingüística latina. Actas del IX Coloquio internacional de lingüística latina (Universidad Autónoma de Madrid, 14-18 de abril de 1997), García Hernández, B. (ed.), vol. 1, Madrid, 397-408.

Hallet, C. H. (2005), The Roman Nude. Heroic Portrait Statuary. 200 BC - AD 300, Oxford.

HILD, J. A. (1904), “Lemures" (s.v.), en Dictionnaire des antiquités grecques et romaines d'après les textes et les monuments, III, Daremberg, Ch. V., Saglio, E. (sous la direction de) Paris.

Hoogma, R. P. (1959), Der Einfluss Vergils auf die Carmina Latina Epigraphica. Eine Studie mit besonderer Berücksichtigung der metrisch-technischen Grundsätze der Entlehnung, Amsterdam.

JAVIER LOMAS, F. (1978), "Tabula Hebana", Habis 9, 323-354.

LiOU-GILle, B. (1980), Culte « hérö̈ques » romains. Les fondateurs, Paris.

LiOU-Gille, B. (1993), "Divinisation des morts dans la Rome ancienne », Revue Belge de philologie et d'histoire 71/1, 107-115.

MAYER, M. (1993), “La presència de Virgili en l'epigrafia d'Hispània. Notes per a un corpus de citacions directes", en Homenatge a Miquel Tarrdell, J. Padró et al. (eds.), Barcelona, 859-869.

MAYER, M. (2007), “Las dedicatorias a miembros de la domus Augusta Julio-Claudia y su soporte: una primera aproximación", en Contributi all'epigrafia d'età 
augustea. Actes de la XIIIe rencontré franco-italienne sur l'épigraphie du monde romain, G. Paci (a cura di), Tivoli, 171-199.

QUiCHERATS, L. (1852), Thesaurus poeticus linguae Latinae, London.

SANTANGELI VALENZANI, R. (2014), "Il sottosuolo come metafora degli inferi”, en La Cloaca Maxima e i sistemi fognari di Roma dall'età antica ad oggi, E. Bianchi (a cura di), Roma, 185-189.

SCHEID, J. (2000), "Les décrets de Pise et le cult des morts", en La commemorazione di Germanico nella documentazione epigrafica. Tabula Hebana e Tabula Siarensis (Convegno Internazionale di Studi, Cassino, 21-24 ottobre 1991), Fraschetti, A. (a cura di), Roma, 131-140.

SCHILling, R. (1980), "La déification à Rome. Tradition latine et interférence grecque", Revue des études latines, 58, 137-152.

SEGENNI, S. (2007), "Calendari e vita municipale (riflessioni su CIL XI A4201421)", Epigraphica 69, 99-115.

SEgEnNI, S. (2011), I Decreta Pisana. Autonomia cittadina e ideologia imperiale nella colonia Opsequens Iulia Pisana, Bari.

Setaioli, A. (1967), “Quisque suos patimur Manes (Verg. Aen. VI, 743)”, Atene e Roma 12, 169-172.

SHERK, R. K. (1970), The Municipal Decrees of the Roman West (Arethusa Monographs), vol. 2, Buffalo.

SuÁreZ DE LA TORRE, E. (2000), "La noción de daimon en la literatura de la Grecia arcaica y clásica", en Seres intermedios. Ángeles, demonios y genios en el mundo mediterráneo, Pérez Jiménez, A., Cruz Andreotti, G. (eds.), Málaga, 47-87.

TANTIMONACO, S. (2015) " "Maschili e femminili», «singolari e plurali». Alcune osservazioni sui Manes a partire dalle fonti epigrafiche", Erga-Logoi, 3 (1), 109-131.

Thomas, R. F., 1987, “Manes” (s.v.), en: Della Corte, F., (a cura di), Enciclopedia Virgiliana, III, Roma, 783-784. 